\title{
Financial Interpretation of Risk Decomposition
}

\author{
Xiaodan Zou \\ Department of Public Economics \\ Xiamen University \\ Xiamen, China \\ e-mail: rabbit.8885@163.com
}

\begin{abstract}
Risk decomposition is very significant for portfolio risk allocation as well as risk monitoring. However, the validity of risk decomposition has long been questioned because it does not have a solid financial interpretation. This paper summarizes and modifies the financial interpretation of risk decomposition in terms of standard deviation, value at risk (VaR) and expected shortfall (ES) from references and performs empirical analysis of each risk measure. The conclusion is that all the risk decomposition in terms of standard deviation, VaR and ES can be interpreted by the corresponding loss contribution.
\end{abstract}

Keywords-financial interpretation; loss contribution; volatility; value at risk; expected shortfall

\section{I.INTRODUCTION}

In modern risk-based investment theory, risk decomposition technique is widely used because this approach puts risk diversification at the core. Under this technique, portfolio risk measure can be mathematically decomposed as marginal risk contribution or percentage risk contribution of each asset. Using it, market researchers can tell what percentage the risk of considered asset contributes to the portfolio risk.

However, this approach is widely disputed because mathematical decomposition does not necessarily have practical significance. To put it in another way, researchers wonder whether each decomposed component has a sound financial interpretation.

Qian (2006) has arrived at the financial interpretation of risk decomposition by building a relationship between loss contribution and percentage risk contribution in terms of volatility and VaR. He further performed an empirical analysis of a balanced portfolio and concluded that risk contribution in terms of volatility and $\mathrm{VaR}$ can be interpreted as expected contribution to potential downside return of the portfolio. As for another important risk measure ES, Boudt, Peterson and Croux (2009) found that the contribution to ES can be interpreted as the expected contribution to portfolio return when the portfolio return is more than or equal to the value of VaR.

However, Qian (2006)'s work on interpreting volatility decomposition is not very convincing: he pointed out three special cases in which the risk contribution can be perfectly interpreted by loss contribution, but the three cases cannot represent all the scenarios; the empirical result was also inconsistent with his theoretical conclusion due to small sample and imperfect assumption. As for the interpretation of ES, Boudt, Peterson and Croux (2009) did not perform empirical analysis to verify their viewpoints.

In this paper, the author provides a new case to supplement the original three cases provided by Qian to make the interpretation of volatility decomposition more widely applied in reality. The author further uses the latest data to perform numerical analysis for volatility, $\mathrm{VaR}$ and ES under this case. The structure of the paper is as follows. Interpretation of risk contribution for volatility, $\mathrm{VaR}$ and ES is presented in Section 2, 3, 4 separately. In each of these sections, numerical analysis is performed following the theoretical part. Finally this paper is concluded in Section 5.

\section{II.PERCENTAGE CONTRIBUTION TO PORTFOLIO VOLATILITY}

Volatility measures the deviation of downside return from the mean. For simplicity, consider only two assets in the portfolio with weights $\omega_{1}$ and $\omega_{2}$ respectively. The return of each asset is denoted by $r_{1}, r_{2}$ with mean $\mu_{1}, \mu_{2}$ and volatility $\sigma_{1}, \sigma_{2}$. The correlation is denoted as $\rho$. Under the assumption of normal distribution, the portfolio mean $\mu$ and standard deviation $\sigma$ is easily obtained by

$$
\begin{gathered}
\mu=\omega_{1} \mathrm{r}_{1}+\omega_{2} \mathrm{r}_{2} \\
\sigma=\sqrt{\omega_{1}^{2} \sigma_{1}^{2}+\omega_{2}^{2} \sigma_{2}^{2}+2 \rho \omega_{1} \omega_{2} \sigma_{1} \sigma_{2}}
\end{gathered}
$$

To involve financial interpretation, we need to know loss contribution, which is defined as $c_{i}=E\left(\omega_{i} r_{i} \mid \omega_{1} r_{1}+\omega_{2} r_{2}=r\right) / r, i=1,2$, namely, the expected contribution from each asset divided by the downside portfolio return.

\section{A. Risk Contribution and Loss Contribution}

According to Euler Decomposition, the portfolio volatility can be decomposed by $\sigma=\omega_{1} \frac{\partial \sigma}{\partial \omega_{1}}+\omega_{2} \frac{\partial \sigma}{\partial \omega_{2}}$, or in percentage form $\sigma / \sigma=\omega_{1} \frac{\partial \sigma}{\partial \omega_{1}} / \sigma+\omega_{2} \frac{\partial \sigma}{\partial \omega_{2}} / \sigma$. Therefore, the percentage contribution of each asset to $\sigma$ can be mathematically defined as

$$
\begin{aligned}
& \mathrm{p}_{1}=\left(\omega_{1} \frac{\partial \sigma}{\partial \omega_{1}}\right) / \sigma=\frac{\omega_{1}^{2} \sigma_{1}^{2}+\rho \omega_{1} \omega_{2} \sigma_{1} \sigma_{2}}{\sigma^{2}} \\
& \mathrm{p}_{1}=\left(\omega_{2} \frac{\partial \sigma}{\partial \omega_{2}}\right) / \sigma=\frac{\omega_{2}^{2} \sigma_{2}^{2}+\rho \omega_{1} \omega_{2} \sigma_{1} \sigma_{2}}{\sigma^{2}}
\end{aligned}
$$


Qian (2006) has proved the following relationship between risk contribution $\mathrm{p}_{\mathrm{i}}$ and loss contribution $\mathrm{c}_{\mathrm{i}}, \mathrm{i}=1,2$ :

$$
\begin{aligned}
c_{1} & =\frac{\omega_{1} \mu_{1}}{r}+p_{1}\left(1-\frac{\omega_{1} \mu_{1}}{r}-\frac{\omega_{2} \mu_{2}}{r}\right) \\
& =p_{1}+\frac{p_{2} \omega_{1} \mu_{1}-p_{1} \omega_{2} \mu_{2}}{r} \\
c_{2} & =\frac{\omega_{2} \mu_{2}}{r}+p_{1}\left(1-\frac{\omega_{1} \mu_{1}}{r}-\frac{\omega_{2} \mu_{2}}{r}\right) \\
& =p_{2}+\frac{p_{1} \omega_{2} \mu_{2}-p_{2} \omega_{1} \mu_{1}}{r}
\end{aligned}
$$

According to this relationship, there are three special cases where the risk contribution equals the loss contribution. Firstly, when $\mu_{1}$ and $\mu_{2}$ are both zero, $\omega_{1} \mu_{1} p_{2}$ equals $\omega_{2} \mu_{2} p_{1}$, implying $c_{1}=p_{1}$ and $c_{2}=p_{2}$. This condition applies to short term return of securities where the expected returns are almost zero. In practice, much risk management analyses are literally done over one-day or one-week scale. Another case is when $\omega_{1}$ or $\omega_{2}$ equals zero, the percentage risk contribution $\mathrm{p}_{1}$ or $\mathrm{p}_{2}$ equals zero, so that $c_{1}=p_{1}, c_{2}=p_{2}$. In practice, this case is barely seen. However, if one of the assets weights very small relatively to the downside portfolio return $r$, the risk contribution can still be considered almost perfectly interpreted by the loss contribution. Finally, if the equation $p_{2} \omega_{1} \mu_{1}=p_{1} \omega_{2} \mu_{2}$ holds or $\frac{\omega_{1} \mu_{1}}{p_{2}}=\frac{\omega_{2} \mu_{2}}{p_{1}}$ holds, the risk contribution is equal to the loss contribution. Actually, this condition is the first order condition of marginal utility for an optimal mean-variance portfolio. In another word, risk contribution perfectly depicts the loss contribution for optimized mean-variance portfolio.

Based on the above fact, Qian (2006) concluded that the volatility decomposition can be financially interpreted by loss contribution. However, as far as the author considered, this conclusion is not very convincing: foremost, except the case when $\mu_{1}=\mu_{2}=0$, chances are limited for other two cases in practice, which is also admitted by Qian (2006); In addition, although the case $\mu_{1}=\mu_{2}=0$ is common, it does not apply to mid-term and long-term horizon. So the author provides another more generalized case: $p_{2} \omega_{1} \mu_{1}-p_{1} \omega_{2} \mu_{2}$ is relatively small to downside portfolio return $r$. This is almost true because $p_{i}, w_{i}, \mu_{i}$ are usually small than one if short sell is not allowed, so their product can be small. More importantly, risk managers pay more attention to large risk, such as the VaR with over $80 \%$ confidence level so that $\mathrm{p}_{2} \omega_{1} \mu_{1}-\mathrm{p}_{1} \omega_{2} \mu_{2}$ looks even smaller compared to the large downside return. Therefore, we conclude that for most practical cases, volatility decomposition can be interpreted by loss contribution.

\section{B. Numeric Analysis}

In this part, the author compares the theoretical predictions with actual results by a portfolio of $40 \% \mathrm{~S} \& \mathrm{P}$ 500 stock and $60 \%$ General Electric Company stock. The author considers monthly return from 1964-01-01 to
2012-01-01 because monthly return has larger sample space than annual one. Table 1 gives the moments and percentage contribution of both stocks.

Table 2 shows the loss contributions predicted by risk contribution together with the realized loss contribution for downside return above $-1 \%$ and in several bins. Actually, the VaR of portfolio downside return at $80 \%$ confidence interval is $-3 \%$, However, the author selects $-1 \%$ instead of $-3 \%$ as the upper bound because $-1 \%$ is already big enough to $p_{2} \omega_{1} \mu_{1}-p_{1} \omega_{2} \mu_{2}=0.0001643$ and it has more sample space for each bin to calculate realized loss contribution than $-3 \%$. Later one can tell that the sample space problem in Qian (2006)'s work has been greatly improved by this way. In table 2, only GE stocks' predicted and realized loss contribution is reported because the percentage contribution of the two stocks adds up to 1 . As the loss contribution can only be predicted by point downside return, the midpoint of each bin is selected as the point return.

TABLE I. MONTHLY RETURN MOMENTS AND RISK CONTRIBUTION

\begin{tabular}{|c|c|c|c|}
\hline & S\&P 500 & GE & Portfolio \\
\hline mean & $0.4857 \%$ & $0.7774 \%$ & $0.6607 \%$ \\
\hline volatility & 0.04411 & 0.07070 & 0.05677 \\
\hline skewness & -0.6628 & -0.3059 & -0.4173 \\
\hline kurtosis & 2.4974 & 1.7045 & 1.9549 \\
\hline p_contrib & 0.2692 & 0.7308 & 1.0000 \\
\hline
\end{tabular}

TABLE II. PREDICTED AND REALIZED LOSS CONTRIBUTION

\begin{tabular}{|c|c|c|c|c|c|}
\hline Return & N & r_c $\mathbf{2}_{2}$ & r_std & P_c $_{2}$ & P_std \\
\hline$(-0.02,-0.01)$ & 37 & 0.8616 & 0.6319 & 0.7418 & 0.7319 \\
\hline$(-0.03,-0.02)$ & 37 & 0.7279 & 0.3080 & 0.7374 & 0.3527 \\
\hline$(-0.04,-0.03)$ & 30 & 0.7784 & 0.2940 & 0.7355 & 0.2519 \\
\hline$(-0.05,-0.04)$ & 25 & 0.7269 & 0.1575 & 0.7345 & 0.1960 \\
\hline$(-0.06,-0.05)$ & 15 & 0.7582 & 0.2196 & 0.7338 & 0.1603 \\
\hline$(-0.07,-0.06)$ & 13 & 0.6230 & 0.1683 & 0.7334 & 0.1357 \\
\hline
\end{tabular}

As the downside return decreases, the predicted loss contribution of GE stock $\left(\mathrm{p}_{-} \mathrm{c}_{2}\right)$ decreases from $74.18 \%$ to $73.34 \%$, approaching its percentage contribution (p_contrib) $73.08 \%$. Table 2 shows the predicted loss contribution agrees with the realized loss contribution $\left(\mathrm{r}_{-} \mathrm{c}_{2}\right)$ in general and perfectly captures the descending trend of the realized values. There seems a large gap between predicted and realized values at the last bin. The reason is that the sample space $\mathrm{N}$ is too small, though it has been enlarged compared to Qian (2006)'s work. Another reason might be the fat tail of the return of the stock, which is inconsistent with the normal distributed assumption. The predicted and realized standard deviations (p_std, r_std) of the percentage contribution are also presented in table 1 that is $s_{i}=\frac{\left|\omega_{1} \omega_{2} \sigma_{1} \sigma_{2} \sqrt{1-\rho^{2}}\right|}{\sigma r}, i=$ 1,2 proved by Qian (2006). On average, they are quite close. In summary, for large downside return above VaR at $80 \%$ confidence level, the risk contribution $73.08 \%$ can be mostly explained by the loss contribution. 


\section{III.PERCENTAGE CONTRIBUTION TO PORTFOLIO VAR}

In this section, the same financial interpretation is extended for $\mathrm{VaR}$ contribution. $\mathrm{VaR}$ is the most popular risk measure in financial institution. It describes the left tail of the return distribution, or more formally, describes a quantile of the projected distribution of downside return over a target horizon. Generally, given a confidence level $\alpha, \mathrm{VaR}_{\alpha}$ is defined by the following equation

$$
\operatorname{VaR}_{\alpha}=\inf \left\{\operatorname{VaR}_{\alpha} \in \mathrm{R} \mid \mathrm{P}\left(\mathrm{r} \leq \operatorname{VaR}_{\alpha}\right) \geq 1-\alpha\right\}
$$

where $\mathrm{r}$ is the downside return. If the return distribution is continuous, $\mathrm{VaR}_{\alpha}$ can be written as $\mathrm{P}(r \leq$ $\left.\operatorname{VaR}_{\alpha}\right)=1-\alpha$.

For non-normal distributed return, VaR can be approximated based on moments of the distribution by Cornish-Fisher expansion as follows

$$
\mathrm{VaR}_{\alpha}=\mu+\widetilde{\mathrm{z}_{\alpha}} \sigma
$$

where $\mu$ is the mean and $\sigma$ is the standard deviation, and

$$
\begin{aligned}
& \widetilde{\mathrm{z}_{\alpha}} \approx \mathrm{z}_{\alpha}+\frac{1}{6}\left(\mathrm{z}_{\alpha}^{2}-1\right) \mathrm{s}+\frac{1}{24}\left(\mathrm{z}_{\alpha}^{3}-3 \mathrm{z}_{\alpha}\right) \mathrm{k} \\
& -\frac{1}{36}\left(2 \mathrm{z}_{\alpha}^{3}-5 \mathrm{z}_{\alpha}\right) \mathrm{s}^{2}
\end{aligned}
$$

where $z_{\alpha}$ is denoted as $\alpha \%$ quantile of standard normal distribution and $\mathrm{s}, \mathrm{k}$ is denoted as skewness and excess kurtosis respectively.

\section{A. Risk contribution and loss contribution}

Since VaR is linear homogeneous function of weights, according to Euler Decomposition, VaR can be mathematically decomposed as

$$
\operatorname{VaR}=\sum_{\mathrm{i}=1}^{\mathrm{N}} \omega_{\mathrm{i}} \frac{\partial \mathrm{VaR}}{\partial \omega_{\mathrm{i}}}
$$

Thus one can define the percentage contribution of asset $i$ to portfolio VaR as $\omega_{\mathrm{i}} \frac{\partial \mathrm{VaR}}{\partial \omega_{\mathrm{i}}} / \mathrm{VaR}$. Hallerbach (2002) realized that the marginal $\mathrm{VaR}$ contribution can be decomposed as sum of conditional expectations of component returns

$$
\operatorname{VaR}=\sum_{i=1}^{N} \omega_{i} E\left(r_{i} \mid \vec{\omega}^{T} \vec{r}=V a R\right)
$$

Comparing the three equations above, Hallerbach (2003) concluded

$$
c_{i}=\frac{E\left(\omega_{i} r_{i} \mid \vec{\omega}^{T} \vec{r}=V a R\right)}{\operatorname{VaR}}=\left(\omega_{i} \frac{\partial V a R}{\partial \omega_{i}}\right) / V a R
$$

meaning that the percentage VaR contribution can be interpreted as expected contribution of the considered asset to the downside portfolio return, namely, the loss contribution, when the portfolio return equals to VaR.

Although contribution to VaR has the similar financial interpretation as volatility both in terms of loss contribution, there are several subtle differences. Firstly, while the interpretation of VaR contribution applies to all cases as long as the downside return is set to be VaR, only in several cases can volatility contribution be perfectly explained by loss contribution. Secondly, when interpreting VaR contribution, the downside return has to be equal to $\mathrm{VaR}$ while the contribution to volatility does not depend on the portfolio return. So in this sense, contribution to $\mathrm{VaR}$ is more restrictive. Thirdly, from equation (12), VaR contribution is always equal to loss contribution as long as the downside return is set to be $\mathrm{VaR}$, which means it is always perfectly explained by loss contribution. For volatility contribution, on the other hand, risk contribution cannot be perfectly interpreted by loss contribution in most time.

\section{B. Numerical analysis}

Jaschke (2002) has shown that the Cornish-Fisher approximation is efficient and accurate enough to obtain VaR. Thus the author uses this method to approximate portfolio VaR and VaR decomposition. Given the same portfolio in section 2.B, percentage contribution from GE stock to different values of portfolio VaR is presented in Table 3. These values are selected to coincide with the midpoints of downside return bins in Table 2 so that we can compare the predicted $\mathrm{c}_{2}$ with the obtained realized $\mathrm{c}_{2}$ of each downside return.

TABLE III. PREDICTED AND REALIZED LOSS CONTRIBUTION

\begin{tabular}{|c|c|c|c|}
\hline Return & CL & P_c 2 & r_c $_{2}$ \\
\hline-0.015 & 0.7050 & 0.8102 & 0.8616 \\
\hline-0.025 & 0.7655 & 0.7752 & 0.7279 \\
\hline-0.035 & 0.8145 & 0.7579 & 0.7784 \\
\hline-0.045 & 0.8535 & 0.7469 & 0.7269 \\
\hline-0.055 & 0.8845 & 0.7388 & 0.7582 \\
\hline-0.065 & 0.9087 & 0.7388 & 0.6230 \\
\hline
\end{tabular}

CL is short for confidence level, obtained by setting $\mathrm{VaR}$ equal to each downside return and used for calculating predicted loss contribution. From table 3, predicted $c_{2}$ agrees with the realized $c_{2}$ on average and has a same descending trend. Compared to volatility contribution, risk contribution in terms of VaR can be more accurately interpreted by realized loss contribution. For instance, when the downside return is -0.015 or within -0.01 and -0.02 , the VaR contribution $(0.8102)$ is better explained than the volatility contribution 0.7308 by realized loss contribution 0.8616 . This is because for each loss contribution, the downside return is equal to VaR so it bears more close relationship with $\mathrm{VaR}$ contribution while volatility contribution is independent of the downside return. Another possible reason is that according to equation (5), as long as $\mathrm{p}_{2} \omega_{1} \mu_{1}-\mathrm{p}_{1} \omega_{2} \mu_{2} \neq$ $0, \mathrm{c}_{2} \approx \mathrm{p}_{2}$, then $\mathrm{p}_{2}$ is only "almost" perfectly explained by $c_{2}$. VaR contribution, however, is always perfectly interpreted by loss contribution $c_{2}$ due to equation (12). On the other hand, it should be noted that while the VaR 
decomposition is better estimated, it does not provide a measure for standard error, which is a disadvantage compared to risk contribution in terms of volatility.

\section{IV.PERCENTAGE CONTRIBUTION TO PORTFOLIO ES}

ES is defined as the expected downside return given that the return falls in the worst $\alpha$ part of the return distribution

$$
\mathrm{ES}_{\alpha}=\left(\mathrm{r} \mid \mathrm{r} \leq \mathrm{VaR}_{\alpha}\right)
$$

In other words, ES is the expected return in the left tail of the return distribution. It gives information about frequency and size of large downside return.

\section{A. Risk contribution and loss contribution}

Scaillet (2002) proved that the first order derivative of the expected shortfall with respect to portfolio allocation is:

$$
\frac{\partial \mathrm{ES}}{\partial \omega_{\mathrm{i}}}=\mathrm{E}\left(\mathrm{r}_{\mathrm{i}} \mid \vec{\omega}^{\mathrm{T}} \overrightarrow{\mathrm{r}}=\mathrm{VaR}\right)
$$

Denote $\vec{\omega}^{T} \vec{r}=r$, Boudt, Peterson and Croux (2009) realized that the above equation is equivalent to

$$
\left(\omega_{\mathrm{i}} \frac{\partial \mathrm{ES}}{\partial \omega_{\mathrm{i}}}\right) / \mathrm{ES}=\mathrm{E}\left(\omega_{\mathrm{i}} \mathrm{r}_{\mathrm{i}} \mid \mathrm{r}<\operatorname{VaR}\right) / \mathrm{E}(\mathrm{r} \mid \mathrm{r} \leq \mathrm{VaR})
$$

So they concluded that percentage contribution to ES can be interpreted as the expected contribution to portfolio return when the downside portfolio return is at most the value of $\mathrm{VaR}$

\section{B. Numerical analysis}

The author uses the Cornish-Fisher approximation to approximate portfolio ES and ES decomposition. Given the same portfolio in Section 2.B, percentage contribution from GE stock to different values of portfolio ES is shown in Table 3. The VaR used for obtaining each ES are selected in the same way as in table 3 .

TABLE IV. PREDICTED AND REALIZED LOSS CONTRIBUTION

\begin{tabular}{|c|c|c|c|c|}
\hline $\mathbf{V a R}_{\boldsymbol{\alpha}}$ & $\mathbf{C L}$ & $\mathbf{N}$ & $\mathbf{r}_{\mathbf{c}} \mathbf{c}_{\mathbf{2}}$ & $\mathbf{P}_{\mathbf{2}} \mathbf{c}_{\mathbf{2}}$ \\
\hline-0.015 & 0.7050 & 184 & 0.7242 & 0.7319 \\
\hline-0.025 & 0.7655 & 141 & 0.7231 & 0.7290 \\
\hline-0.035 & 0.8145 & 112 & 0.7186 & 0.7272 \\
\hline-0.045 & 0.8535 & 87 & 0.7133 & 0.7258 \\
\hline-0.055 & 0.8845 & 59 & 0.7084 & 0.7235 \\
\hline-0.065 & 0.9087 & 50 & 0.7125 & 0.7189 \\
\hline
\end{tabular}

$\mathrm{N}$ is the sample space of the range where the downside return is smaller than each VaR. A few observations can be made from table 4. Predicted loss contribution generally agrees with the realized $c_{2}$ and has the same descending trend as the realized one. Similar to the VaR contribution, ES contribution is perfectly interpreted by the corresponding loss contribution while no standard error is given in the prediction.

\section{V.CONCLUSION}

Both theoretical deduction and empirical evidences illustrate that risk decomposition has a valid financial interpretation in terms of loss contribution. When the considered return is assumed to be normal, contribution to portfolio volatility is easy to obtain and often approximately depicts the loss contribution in practice. Risk contribution in terms of $\mathrm{VaR}$ and ES, on the other hand, is more precisely interpreted by the loss contribution and the interpretation is universally applicable.

\section{REFERENCE}

[1] Qian, E.E. (2006). On the Financial Interpretation of Risk Contribution: Risk Budgets Do Add Up, Journal of Investment Management, 64, 527-560

[2] Boudt, K., B. Peterson and C. Croux (2008). Estimation and Decomposition of Downside Risk for Portfolios with Non-Normal Returns, Journal of Risk, 64, 527-560

[3] Scaillet, O. (2002). Nonparametric estimation and sensitivity analysis of expected shortfall, Mathematical Finance 14(1), 74-86

[4] Hallerbach, Winfried G. (2003), Decomposing Portfolio Value-atRisk: A General Analysis, Journal of Risk. Volume 5 / Number 2

[5] Jaschke, Stefan R. (2002), The Cornish-Fisher Expansion in the Context of Delta-Gamma-Normal A 